\title{
A4GALT mediated the glycosylation of B7-H3 in human colorectal cancer cell lines
}

\section{Fuchao Li}

Nanjing Medical University

\section{Dan Han ( $\nabla$ handanjelly@126.com )}

Nanjing Drum Tower Hospital: Nanjing University Medical School Affiliated Nanjing Drum Tower Hospital https://orcid.org/0000-0003-2183-2503

\section{Mengxi Chen}

Soochow University

Jingmei Li

Soochow University

\section{Li Yang}

Nanjing Drum Tower Hospital: Nanjing University Medical School Affiliated Nanjing Drum Tower Hospital

\section{Weipeng Wang}

Soochow University

Chenggong Yu

Nanjing Drum Tower Hospital: Nanjing University Medical School Affiliated Nanjing Drum Tower Hospital

\section{Research Article}

Keywords: A4GALT, B7-H3, glycosylation, GALT, galactose

Posted Date: February 7th, 2022

DOI: https://doi.org/10.21203/rs.3.rs-1197553/v2

License: (a) (i) This work is licensed under a Creative Commons Attribution 4.0 International License. Read Full License 


\section{A4GALT mediated the glycosylation of B7-H3 in human colorectal cancer cell lines}

Fuchao Li ${ }^{1,2 \#}$, Dan $\mathrm{Han}^{3 \#}$, Mengxi Chen ${ }^{4 \#}$, Jingmei $\mathrm{Li}^{4}$, Li Yang ${ }^{2}$, Weipeng Wang $4 *$, Chenggong Yu ${ }^{1 *}$

1 Department of Gastroenterology, Nanjing Drum Tower Hospital Clinical College of Nanjing Medical University, Nanjing, China.

2 Department of Geriatrics, Nanjing Drum Tower Hospital, The Affiliated Hospital of Nanjing University Medical School, Nanjing, China

3 Department of Pharmacy, Nanjing Drum Tower Hospital, The Affiliated Hospital of Nanjing University Medical School, Nanjing, China

4 Center for Drug Metabolism and Pharmacokinetics, College of Pharmaceutical Sciences, Soochow University, Suzhou 215123, China.

* Correspondence authors: Weipeng Wang, Yunxuan Building \#1339, College of Pharmaceutical Sciences, Soochow University, Wenjing Road, Suzhou Industrial Park, Suzhou 215123, China. E-mail: wangweipeng@suda.edu.cn; Tel/Fax: $+86-512-65883761$.

\# These authors contributed equally to this work. 


\begin{abstract}
\end{abstract}
B7-H3 is one of the most important members of the B7/CD28 family, and its expression level is abnormally high in a variety of tumors. B7-H3 inhibits $\mathrm{T}$ cell activation via binding to the corresponding receptors on $\mathrm{T}$ cells, thereby mediating tumor immune escape. Glycosylation is a common post-translational modification of proteins, which plays an essential role in protein expression patterns and biological functions. Current evidence has shown that the abnormal glycosylation of B7-H3 in tumors is of great significance for protein expression and ligand-receptor binding. Therefore, in-depth exploration of the underlying mechanism of glycosylation modification of B7-H3 is expected to provide new insights for tumor immunotherapy. To investigate the underlying mechanism of glycosyltransferase-mediated glycosylation of B7-H3. Firstly, the CHIPBase database was used to screen glycosyltransferases with a high correlation with the protein expression of B7-H3. Then their siRNAs were designed and synthesized to transfect into cells, and the western blotting assay was performed for further screening. Secondly, the siRNA and overexpression plasmid of the screened glycosyltransferase were respectively transfected into cells to verify the effect on the expression level of B7-H3. Thirdly, the effect of glycosyltransferase on the expression level of B7-H3 was explored by changing its substrate level. Finally, the co-immunoprecipitation experiment was conducted to verify whether protein binding existed between B7-H3 and the glycosyltransferase. The glycosyltransferase called A4GALT had the highest correlation with the protein expression of B7-H3. After knocking down or overexpressing A4GALT, the protein expression level of B7-H3 both changed significantly. When knocked down GALT to reduce the galactosyl donors, B7-H3 was significantly down-regulated. And B7-H3 was up-regulated while increasing the galactose in the medium. In addition, the Co-immunoprecipitation experiment proved that there was protein binding between B7-H3 and A4GALT. A4GALT can positively regulate the expression of $\mathrm{B} 7-\mathrm{H} 3$, and changing the level of galactosyl donors can also positively regulate the expression of $\mathrm{B} 7-\mathrm{H} 3$. There is a protein 
interaction between A4GALT and B7-H3.

Keywords: A4GALT; B7-H3; glycosylation; GALT; galactose 


\section{Introduction}

In recent years, the incidence and mortality of tumors have been rising year by year ${ }^{[1]}$. At present, surgery, chemotherapy, and radiotherapy are the traditional therapies of tumors. Although the development of tumor immunotherapy has partly overcome the shortcomings of conventional therapies, it still has clinical limitations. Therefore, it is essential to find key therapeutic targets to develop new approaches for tumor treatment.

$\mathrm{T}$ cell activation requires dual signal stimulation. The first signal is initiated by the $\mathrm{T}$ cell antigen receptor (TCR) recognizing the antigen peptide-MHC complex on the surface of the antigen-presenting cell (APC) cell. The second signal is initiated by the $\mathrm{T}$ cell, and it is provided by mutual recognition with co-signal molecules on the surface of APC ${ }^{[2]}$. The second signal is the key to the complete activation of $\mathrm{T}$ cells, and B7/CD28 is one of the important families involved in mediating this signal. The B7 molecules on the surface of APC and the CD28 molecules on the surface of T cells mediate the second signal through ligand-receptor binding. This signal includes costimulatory and co-inhibitory signals, stimulating and inhibiting $\mathrm{T}$ cell activation, respectively ${ }^{[3]}$. It is worth mentioning that the therapeutic strategy of blocking the co-inhibitory signal has achieved a significant breakthrough in tumor immunotherapy. The combination of B7-1 and CTLA4, as well as PD-1 and PD-L1, produce the second signals to inhibit $\mathrm{T}$ cell activation ${ }^{[4]}$, related target drugs include Ipilimumab, Yervoy, Pembrolizumab, Nivolumab, Atezolizumab, Avelumab, and Durvalumab, etc. have been marketed ${ }^{[5-7]}$.

B7-H3 is a crucial costimulatory/coinhibitory molecule in the B7 family, and its abnormally high expression results in the imbalance of $\mathrm{T}$ cell immune response, enabling tumor immune escape. Chen JT et al. found that B7-H3 was significantly overexpressed in oral squamous carcinoma. Moreover, B7-H3 was closely related to tumor size, clinical stage, and low survival rate of patients with oral squamous carcinoma. In addition, the proliferation of tumor cells is inhibited after knocking down B7-H3 and then recovered after B7-H3 restoration ${ }^{[8]}$. 
Glycosylation of proteins refers to the process in which glycosyltransferases take nucleoside sugars as sugar donors and link them to amino acid residues of proteins to form glycosidic bonds. As an essential post-translational modification of proteins, glycosylation is ubiquitous in more than $50 \%$ of eukaryotic organisms. Glycosylation is of great importance to protein stability, folding and biological functions ${ }^{[9]}$. This modification occurs at various stages of the cell life cycle and can regulate the expression and function of proteins. Studies have confirmed that the glycosylation of tumor-associated proteins is closely related to the occurrence and development of tumors ${ }^{[10]}$. Current studies showed that the B7/CD28 family glycosylation mainly affects protein stability, expression, and receptor-ligand binding ability. For example, glycosylation has a positive regulation on the expression of PD-1 and B7-DC ${ }^{[11,12]}$ and the binding of PD-L1 to the receptor-ligand of B7-DC ${ }^{[13,14]}$. Therefore, clarifying the glycosylation mechanism of the B7-H3 is conducive to the development of new approaches for tumor immunotherapy that target glycosylation.

\section{Materials and methods}

\subsection{Database screening}

The CHIPBase database (http://deepbase.sysu.edu.cn/chipbase/) was used to find the correlation between the expression of B7-H3 protein and different types of glycosyltransferases, and the correlation coefficients were listed in Supplementary Table 1. The screening was conducted according to the correlation coefficient for subsequent research.

\subsection{Cell culture}

Human colorectal cancer cell lines HCT-116 and SW480 (ATCC, USA) were cultured in the DMEM medium containing 10\% fetal bovine serum (FBS) in an incubator with $5 \% \mathrm{CO}_{2}$ at $37^{\circ} \mathrm{C}$. Cells in the logarithmic period were collected for the following experiments.

\subsection{Cell transfection}


Lipofectamine 2000 (Invitrogen, MA, United States) was used to transfect siRNAs and overexpression plasmids (GENEWIZ, Suzhou, China) into cells. Cells were collected for RNA analysis after 48 hours and protein detection after 72 hours.

\subsection{Reverse transcription PCR (RT-PCR) and quantitative PCR (qPCR)}

Total RNA was extracted using the RNAiso Plus (TaKaRa, Beijing, China), and the concentration was measured using the NanoDrop ND-2000 spectrophotometer (Thermo, MA, United States). After reverse transcription, cDNAs, designed primers (Table S4), and the SYBR Green Supermix (Bio-Rad, CA, United States) were prepared for thermal reactions using the CFX96 Touch ${ }^{\mathrm{TM}}$ real-time PCR system (Bio-Rad). GAPDH was used as an endogenous quantitative control. The relative mRNA expression levels were calculated using the $2^{-\triangle \Delta C q}$ method.

\subsection{Western blotting}

Total protein was extracted by RIPA lysis buffer, and the concentration was measured using the BCA Protein Assay Kit (Thermo). The protein was loaded after denaturation, separated by $10 \%$ SDS-PAGE, and transferred to the polyvinylidene fluoride (PVDF) membrane (Millipore, Germany). After blocking with 5\% skimmed milk in TBST at room temperature for 1.5 hours, the membrane was washed three times with TBST and incubated with the primary antibody solution of the target protein overnight at $4^{\circ} \mathrm{C}$. The membrane was washed three times with TBST and incubated with a secondary antibody (Beyotime) conjugated with horseradish peroxidase corresponding to the primary antibody for $1.5 \mathrm{~h}$ at room temperature. Finally, visual analysis was performed in ChemiDoc ${ }^{\mathrm{TM}}$ MP Imaging System (Bio-Rad).

\subsection{Co-immunoprecipitation}

The cell culture plate was taken out from the cell incubator and washed 3 times with pre-cooled sterile PBS buffer. RIPA lysate was added and placed on ice for 20 min before being transferred to a pre-cooled tube. Protein lysate was collected by centrifugation at $12000 \mathrm{~g}$ at $4^{\circ} \mathrm{C}$. An appropriate amount of protein lysate was taken, and $20 \mu \mathrm{L}$ agarose beads were added. After $30 \mathrm{~min}$ incubation at $4^{\circ} \mathrm{C}$, the 
supernatant was collected by centrifugation at $12000 \mathrm{~g}$ at $4^{\circ} \mathrm{C}$. Diluted with PBS into $500 \mathrm{mg} / \mathrm{mL}$ protein solution, $10 \mu \mathrm{L}$ GAPDH antibody or B7-H3 antibody solution was added and incubated at $4{ }^{\circ} \mathrm{C}$ for $1 \mathrm{~h}$. Then $20 \mu \mathrm{L}$ agarose beads were added and incubated at $4^{\circ} \mathrm{C}$ overnight. The beads were collected by centrifugation and washed 5 times with PBS buffer. $40 \mu \mathrm{L}$ PBS and $8 \mu \mathrm{L} 6 \times$ sample loading buffer were added into the collected samples, boiled at $95^{\circ} \mathrm{C}$ for $5 \mathrm{~min}$, and then the samples were performed for western blotting detection.

\subsection{Statistical analysis}

Data were obtained from three triplicate experiments and expressed as mean \pm standard deviation. Differences between groups were compared by the Student's t-test. The p-values $<0.05$ were considered statistically significant.

\section{Results}

\subsection{Correlation between glycosyltransferases and B7-H3}

The correlations between glycosyltransferases and B7-H3 expression were searched in the CHIPBase database, and the results were shown in Table S1. A total of 14 kinds of glycosyltransferases with high correlation were preliminarily screened out. Their names, correlation coefficients (r-value), and p-value were listed in Table S2.

\subsection{Regulation of B7-H3 expression by glycosyltransferase}

We further synthesized siRNAs targeting the 14 types of glycosyltransferases, and the sequences were shown in Table S3. The qPCR experiments verified the inhibition efficiency of siRNAs, and the results were shown in Fig. S1, indicating that the above siRNAs effectively inhibited the expression of target genes at the mRNA level. Next, the above siRNAs were respectively transfected into HCT-116 cells for $72 \mathrm{~h}$, and the protein expression level of B7-H3 was detected by western blotting. As shown in Fig. 1, the expression level of B7-H3 was significantly downregulated after knocking down FUT7, FUT8, ST3GAL1, ST3GAL2, and A4GALT. Afterwards, we investigated the concentration dependence of the above 5 
siRNAs for further screening. The concentration gradients were 25, 50, and $100 \mu \mathrm{M}$. The results of western blotting were shown in Fig. 2. It could be seen that A4GALT had the most significant impact on the protein expression of $\mathrm{B} 7-\mathrm{H} 3$, preliminarily suggesting its vital function in the glycosylation of B7-H3.

\subsection{A4GALT-mediated regulation of the B7-H3 expression}

To in-depth explore the regulatory effect of A4GALT on B7-H3, we next examined the protein level of B7-H3 in cells with knocking down or overexpressing A4GALT, respectively. First of all, the efficacy of the A4GALT vector was verified in HCT-116 cells by western blotting (Fig. S2). The results showed that the overexpression plasmid of A4GALT was successfully constructed. Then the expression of B7-H3 was detected after knocking down or overexpressing A4GALT. The results demonstrated in Fig. 3 indicated that the expression of B7-H3 was significantly up-regulated with the overexpression of A4GALT. In addition, the expression of B7-H3 was significantly down-regulated after the transfection of siRNA to knock down A4GALT. Furthermore, SW480 cells were selected to verify the experimental results. As shown in Fig. 3, results showed that A4GALT also regulated the expression of $\mathrm{B} 7-\mathrm{H} 3$ in SW480 cells, which was consistent with HCT-116 cells. As expected, these results proved that A4GALT had a positive regulation of the protein expression of $\mathrm{B} 7-\mathrm{H} 3$.

\subsection{Regulation of the B7-H3 expression by galactose}

A4GALT is a galactosyltransferase, and its physiological function depends on the level of the substrate. Therefore, the influence of A4GALT on the expression level of B7-H3 can be further verified by changing the content of galactose donors.

In the process of glycosylation, the sugar donor is nucleoside sugar rather than free galactose, and guanosine diphosphate galactose (UDP-Gal) is transferred by galactosyltransferase. According to the Leloir pathway, galactose-1-phosphate uridine transferase (GALT) is the key enzyme for the production of UDP-Gal. Therefore, we designed and synthesized siRNA of GALT (the sequence was shown in Table S2), transfected it into cells to reduce galactose donors, and then detected 
the expression of B7-H3 by western blotting. In addition, $120 \mathrm{mM}$ galactose was added to the medium, and the same amount of trehalose was added as a control. The expression level of B7-H3 was also detected. It could be seen from the results that the expression of B7-H3 was down-regulated after reducing the galactose donors and up-regulated after increasing the galactose donors (Fig.4). The above results confirmed that A4GALT had a significant effect on the protein expression level of B7-H3.

\subsection{Protein interaction between A4GALT and B7-H3}

Glycosylation is performed by glycosyltransferases which transfer the corresponding glycosyl donors and attach them to the amino acid residues of the proteins. There is an interaction between the glycosyltransferase and the modified protein in this process. To further confirm the A4GALT-mediated regulation of B7-H3 expression, we conducted co-immunoprecipitation experiments to verify the interaction between A4GALT and B7-H3. B7-H3 antibody was used to precipitate B7-H3, and then we performed the western blotting assay to detect whether A4GALT was also co-precipitated.

The results in Fig. 4 showed that a conspicuous band of A4GALT could be detected when B7-H3 was precipitated. Meanwhile, a shallow A4GALT protein band was seen in blank and negative control groups, which may be caused by inadequate cleaning of agarose beads. In addition, no obvious A4GALT protein bands were detected in the input group, possibly resulting from the extremely deficient protein expression level of A4GALT in HCT-116 cells. In conclusion, the experimental results showed a protein interaction between B7-H3 and A4GALT, proving that A4GALT significantly regulated the protein expression of B7-H3.

\section{Discussion}

B7-H3 is one of the negative costimulatory molecules in the B7 family, and its abnormal expression disrupts the balance of $\mathrm{T}$ cell immune response, thus mediating tumor immune escape. Numerous studies have shown that B7-H3 protein is 
abnormally high expressed in colorectal cancer ${ }^{[15]}$, prostate cancer ${ }^{[16]}$, pancreatic cancer ${ }^{[17]}$, squamous cell carcinoma ${ }^{[18]}$, non-small cell lung cancer ${ }^{[19]}$, gastric cancer $^{[20,21]}$ and other tumors, and its expression has significant clinical significance. A study shows that the expression level of B7-H3 in colorectal cancer is negatively correlated with tumor size and prognosis ${ }^{[15]}$. Moreover, the positive expression rate of B7-H3 in ovarian cancer is more than $90 \%$ and is significantly associated with tumor stage, high recurrence rate, and low survival rate ${ }^{[22]}$. In addition, down-regulation of B7-H3 expression can reduce tumor cell adhesion and mobility in melanoma and breast cancer ${ }^{[23]}$.

As an essential immunomodulatory molecule, B7-H3 inhibits the proliferation of CD4+ and CD8+T cells and also inhibits the production of IL-2 and IFN-r. Blocking the binding of $\mathrm{B} 7-\mathrm{H} 3$ with antagonists can promote the expansion of $\mathrm{T}$ cells and improve the expression level of IL-2 ${ }^{[24,25]}$. In addition to the above inhibitory effect on T cells, B7-H3 also inhibits the activity of natural killer (NK) cells. 4IG-B7-H3 is the main existing form of B7-H3, which inhibits NK cell-mediated cell killing after binding to the surface receptors of NK cells ${ }^{[26,27]}$. The above studies indicate that B7-H3 has a definite inhibitory effect on T cells and NK cells and thus has an adverse impact on tumor immune monitoring. Tumor immune surveillance is considered to be an inhibition of cancer cells and maintain the steady-state of the host protection process, cancerous cells can be immune effector cells to clear, but the abnormal expression and glycosylation of negative synergy stimulus molecules can reduce the immunogenicity of cancerous cells, causing the cancer cells can't be immune effector cells to identify and eliminate.

Glycosylation is a process of glycoside connection between sugars and other sugars, proteins or lipids, and is a key regulatory mechanism involved in various physiological and pathological processes ${ }^{[29,30]}$. Glycosylation improves protein heterogeneity and diversity of physiological functions, thus affecting tumor cell metabolism, adhesion, cell-matrix interaction, signal transduction, and immune surveillance ${ }^{[31]}$. There are extensive changes in the types and levels of glycosylation 
in tumor cells ${ }^{[32]}$. Some studies have shown that $7-\mathrm{H} 3$ also has abnormal glycosylation in head and neck squamous cell carcinoma and oral squamous cell carcinoma $^{[8,28]}$.

In recent years, glycobiology has played an increasingly important role in tumor research, which provides a new direction for studying tumor therapeutic targets. In this study, glycosyltransferase called A4GALT, which significantly affects the protein expression of $\mathrm{B} 7-\mathrm{H} 3$, was screened out through the combination of virtual database screening and experimental verification. A4GALT (also known as Gb3/CD77 synthase) can transfer galactose residues to the receptors, and it is a crucial enzyme in the biosynthesis of sphingolipid (GSLs) ${ }^{[33]}$. The enzyme encoded by the A4GALT gene catalyzes the synthesis of P1 and PK antigens and is also widely used in P1PK blood group systematic typing due to its gene polymorphism [34]. In addition, studies have reported that knocking down A4GALT can significantly inhibit contact inhibition, and exogenous adding A4GALT can inhibit the proliferation of low-density cells, suggesting that A4GALT is involved in the 'contact inhibition' of cell growth ${ }^{[35]}$. Other studies have found that A4GALT-mediated GSLs can enhance the metastasis ability of mesenchymal and epithelial cancer cells, and the loss of A4GALT mediated by CRISPR-Cas9 can weaken galactose induced epithelial-mesenchymal transformation (EMT) ${ }^{[36]}$.

In general, the glycosylation pathway in cancer cells changes a lot, resulting in variation of cellular glycan expression. Firstly, fluctuation in glycosyltransferase expression can be attributed to regulation of glycosyltransferase at the transcriptional level ${ }^{[37,38]}$, chaperone dysfunction ${ }^{[39]}$, and glycosidase inactivation ${ }^{[40]}$. Secondly, the changes in glycan expression may be due to changes in the tertiary conformation of the peptide backbone and the new glycan chain. Furthermore, this difference may be due to the variability of different receptor substrates and the availability and the abundance of oligonucleotide donors and cofactors ${ }^{[41]}$. Finally, the changes in glycosyltransferase expression may be caused by the expression and localization of related glycosyltransferases in the Golgi apparatus ${ }^{[42]}$. 
This study confirmed that the expression of B7-H3 was up-regulated after overexpressing A4GALT, and the expression of B7-H3 was down-regulated after knocking down A4GALT, suggesting that A4GALT positively regulated the expression of $\mathrm{B} 7-\mathrm{H} 3$. At the same time, this study found that elevating the concentration of galactose also positively regulated the B7-H3 expression.

This study also verified the protein interaction between A4GALT and B7-H3 through the co-immunoprecipitation experiment, confirming that A4GALT had an essential role in regulating $\mathrm{B} 7-\mathrm{H} 3$. In the co-immunoprecipitation experiment, A4GALT protein bands also appeared in the blank and negative control groups due to the poor cleaning of agarose beads. Impurity bands may be eliminated by changing the reagent used to wash the beads, increasing the number of washes, and reducing the total protein concentration. In addition, the protein band of A4GALT in the input group was not prominent, which may be due to the low expression of A4GALT in HCT-116 cells.

Taken together, this study showed that A4GALT was involved in the glycosylation of B7-H3 and positively regulated the protein expression of B7-H3. Therefore, A4GALT as a target to inhibit the expression of B7-H3 was expected to become a new approach for tumor therapy.

\section{Conflict of Interest Statement}

The authors have no conflicts of interest to declare.

Ethical approval This article does not contain any studies with human participants or animals performed by any of the authors. 


\section{References}

1. Chen, W., et al., Cancer statistics in China, 2015. CA Cancer J Clin, 2016. 66(2): p. 115-32.

2. Chen, L. and D.B. Flies, Molecular mechanisms of $T$ cell co-stimulation and co-inhibition. Nat Rev Immunol, 2013. 13(4): p. 227-42.

3. Zhang, Q. and D.A. Vignali, Co-stimulatory and Co-inhibitory Pathways in Autoimmunity. Immunity, 2016. 44(5): p. 1034-51.

4. Goodman, A., S.P. Patel, and R. Kurzrock, PD-1-PD-L1 immune-checkpoint blockade in B-cell lymphomas. Nat Rev Clin Oncol, 2017. 14(4): p. 203-220.

5. Hodi, F.S., et al., Improved survival with ipilimumab in patients with metastatic melanoma. N Engl J Med, 2010. 363(8): p. 711-23.

6. Topalian, S.L., et al., Safety, activity, and immune correlates of anti-PD-1 antibody in cancer. N Engl J Med, 2012. 366(26): p. 2443-54.

7. Robert, C., et al., Pembrolizumab versus Ipilimumab in Advanced Melanoma. N Engl J Med, 2015. 372(26): p. 2521-32.

8. Chen, J.T., et al., Glycoprotein B7-H3 overexpression and aberrant glycosylation in oral cancer and immune response. Proc Natl Acad Sci U S A, 2015. 112(42): p. 13057-62.

9. Dube, D.H. and C.R. Bertozzi, Glycans in cancer and inflammation--potential for therapeutics and diagnostics. Nat Rev Drug Discov, 2005. 4(6): p. 477-88.

10. Stowell, S.R., T. Ju, and R.D. Cummings, Protein glycosylation in cancer. Annu Rev Pathol, 2015. 10: p. 473-510.

11. Li, C.W., et al., Glycosylation and stabilization of programmed death ligand-1 suppresses T-cell activity. Nat Commun, 2016. 7: p. 12632.

12. Wang, H., et al., PD-L2 expression in colorectal cancer: Independent prognostic effect and targetability by deglycosylation. Oncoimmunology, 2017. 6(7): p. e1327494.

13. Cheng, X., et al., Structure and interactions of the human programmed cell death 1 receptor. J Biol Chem, 2013. 288(17): p. 11771-85. 
14. Li, C.W., et al., Eradication of Triple-Negative Breast Cancer Cells by Targeting Glycosylated PD-L1. Cancer Cell, 2018. 33(2): p. 187-201 e10.

15. Zhang, C., et al., B7-H3 is spliced by SRSF3 in colorectal cancer. Cancer Immunol Immunother, 2021. 70(2): p. 311-321.

16. Benzon, B., et al., Correlation of B7-H3 with androgen receptor, immune pathways and poor outcome in prostate cancer: an expression-based analysis. Prostate Cancer Prostatic Dis, 2017. 20(1): p. 28-35.

17. Loos, M., et al., Expression of the costimulatory molecule B7-H3 is associated with prolonged survival in human pancreatic cancer. BMC Cancer, 2009. 9: p. 463.

18. Zhang, S.S., et al., Expression levels of B7-H3 and TLT-2 in human oral squamous cell carcinoma. Oncol Lett, 2015. 10(2): p. 1063-1068.

19. Xu, Y.H., et al., B7-H3 and CD133 expression in non-small cell lung cancer and correlation with clinicopathologic factors and prognosis. Saudi Med J, 2010. 31(9): p. 980-6.

20. Arigami, T., et al., B7-H3 expression in gastric cancer: a novel molecular blood marker for detecting circulating tumor cells. Cancer Sci, 2011. 102(5): p. 1019-24.

21. Li, Y., et al., B7-H3 promotes gastric cancer cell migration and invasion. Oncotarget, 2017. 8(42): p. 71725-71735.

22. Zhang, J., et al., B7-H3 is related to tumor progression in ovarian cancer. Oncol Rep, 2017. 38(4): p. 2426-2434.

23. Chen, Y.W., C. Tekle, and O. Fodstad, The immunoregulatory protein human $\mathrm{B} 7 \mathrm{H} 3$ is a tumor-associated antigen that regulates tumor cell migration and invasion. Curr Cancer Drug Targets, 2008. 8(5): p. 404-13.

24. Prasad, D.V., et al., Murine B7-H3 is a negative regulator of T cells. J Immunol, 2004. 173(4): p. 2500-6.

25. Suh, W.K., et al., The B7 family member B7-H3 preferentially down-regulates T helper type 1-mediated immune responses. Nat Immunol, 2003. 4(9): p. 
899-906.

26. Steinberger, P., et al., Molecular characterization of human 4Ig-B7-H3, a member of the B7 family with four Ig-like domains. J Immunol, 2004. 172(4): p. 2352-9.

27. Castriconi, R., et al., Identification of $4 \mathrm{Ig}-\mathrm{B} 7-\mathrm{H} 3$ as a neuroblastoma-associated molecule that exerts a protective role from an NK cell-mediated lysis. Proc Natl Acad Sci U S A, 2004. 101(34): p. 12640-5.

28. Liao, C., et al., Changes in Protein Glycosylation in Head and Neck Squamous Cell Carcinoma. J Cancer, 2021. 12(5): p. 1455-1466.

29. Moremen, K.W., M. Tiemeyer, and A.V. Nairn, Vertebrate protein glycosylation: diversity, synthesis, and function. Nat Rev Mol Cell Biol, 2012. 13(7): p. 448-62.

30. Fuster, M.M. and J.D. Esko, The sweet and sour of cancer: glycans as novel therapeutic targets. Nat Rev Cancer, 2005. 5(7): p. 526-42.

31. Pinho, S.S. and C.A. Reis, Glycosylation in cancer: mechanisms and clinical implications. Nat Rev Cancer, 2015. 15(9): p. 540-55.

32. Corfield, A.P. and M. Berry, Glycan variation and evolution in the eukaryotes. Trends Biochem Sci, 2015. 40(7): p. 351-9.

33. Szymczak-Kulus, K., et al., Human Gb3/CD77 synthase produces P1 glycotope-capped N-glycans, which mediate Shiga toxin one but not Shiga toxin 2 cell entry. J Biol Chem, 2021. 296: p. 100299.

34. Chen, Q., et al., Novel A4GALT gene variation with rare p phenotype in a compound heterozygous Chinese individual. Transfusion, 2021. 61(1): p. E5-E6.

35. Huang, X., et al., Functional role of glycosphingolipids in contact inhibition of growth in a human mammary epithelial cell line. FEBS Lett, 2017. 591(13): p. 1918-1928.

36. Jacob, F., et al., Transition of Mesenchymal and Epithelial Cancer Cells Depends on alpha1-4 Galactosyltransferase-Mediated Glycosphingolipids. 
Cancer Res, 2018. 78(11): p. 2952-2965.

37. Kannagi, R., et al., Current relevance of incomplete synthesis and neo-synthesis for cancer-associated alteration of carbohydrate determinants--Hakomori's concepts revisited. Biochim Biophys Acta, 2008. 1780(3): p. 525-31.

38. Pinho, S.S., et al., Loss and recovery of Mgat3 and GnT-III Mediated E-cadherin $\mathrm{N}$-glycosylation is a mechanism involved in epithelial-mesenchymal-epithelial transitions. PLoS One, 2012. 7(3): p. e33191.

39. Aryal, R.P., T. Ju, and R.D. Cummings, The endoplasmic reticulum chaperone Cosmc directly promotes in vitro folding of T-synthase. J Biol Chem, 2010. 285(4): p. 2456-62.

40. Kakugawa, Y., et al., Up-regulation of plasma membrane-associated ganglioside sialidase (Neu3) in human colon cancer and its involvement in apoptosis suppression. Proc Natl Acad Sci U S A, 2002. 99(16): p. 10718-23.

41. Kumamoto, K., et al., Increased expression of UDP-galactose transporter messenger RNA in human colon cancer tissues and its implication in synthesis of Thomsen-Friedenreich antigen and sialyl Lewis A/X determinants. Cancer Res, 2001. 61(11): p. 4620-7.

42. Gill, D.J., et al., Regulation of O-glycosylation through Golgi-to-ER relocation of initiation enzymes. J Cell Biol, 2010. 189(5): p. 843-58. 


\section{Figure legends}

Figure 1. The protein expression of B7-H3 in HCT-116 cells transfected with siRNAs targeting the 14 types of glycosyltransferases.

Figure 2. The regulation of B7-H3 expression by glycosyltransferase The Protein expression levels of B7-H3 in HCT-116 cells after transfecting with ST3GAL1 siRNA (A), ST3GAL2 siRNA (B), FUT7 siRNA (C), FUT8 siRNA (D), and A4GALT siRNA (E); The relative expression of B7-H3 after transfection of siRNAs (F).

Figure 3. The A4GALT-mediated regulation of the protein expression of $\mathrm{B} 7-\mathrm{H} 3$ in HCT-116 and SW480 cells.

Figure 4. The regulatory effect of the B7-H3 expression by galactose.

Figure 5. The protein-protein interaction between B7-H3 and A4GALT. 
Figure 1

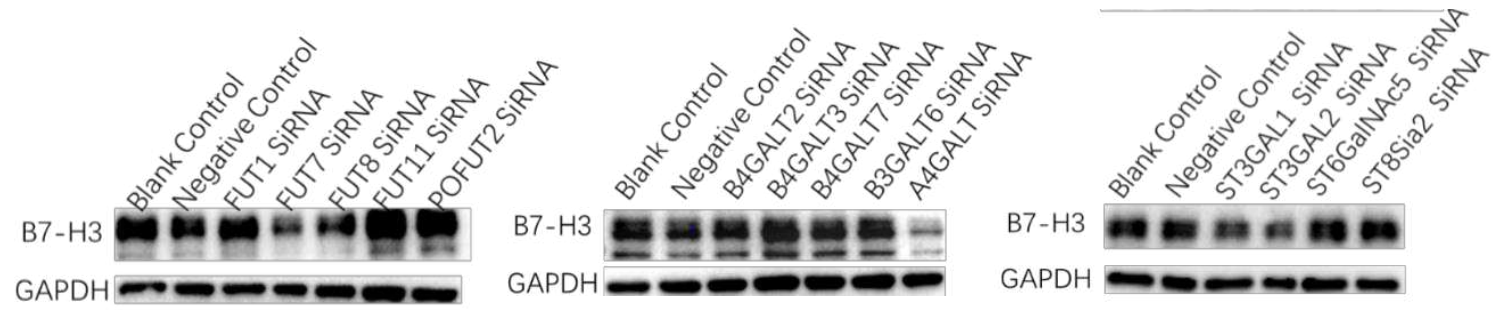




\section{Figure 2}
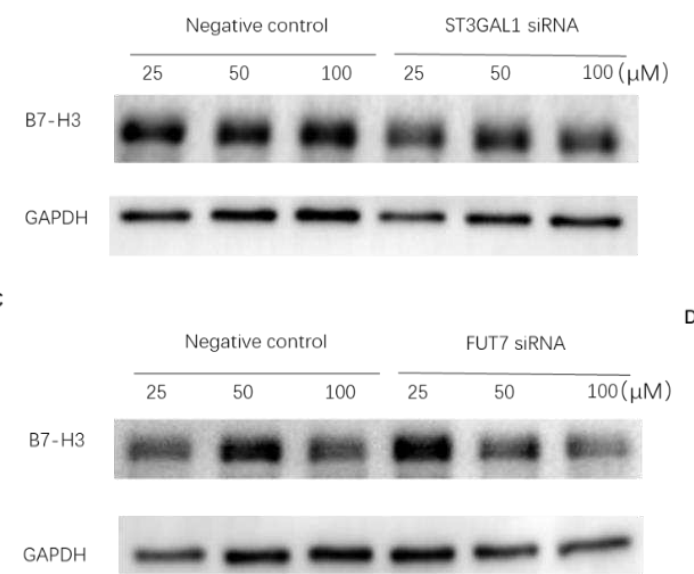

E

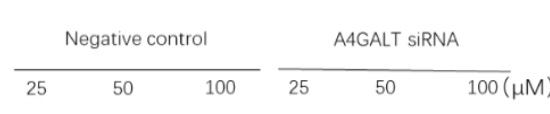

B7-H3

GAPDH
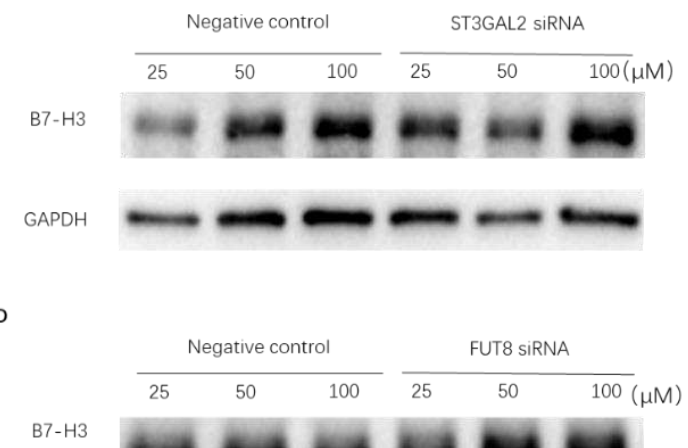

$\mathrm{B} 7-\mathrm{H} 3$

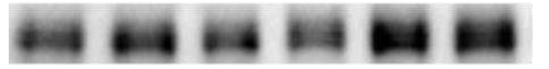

GAPDH
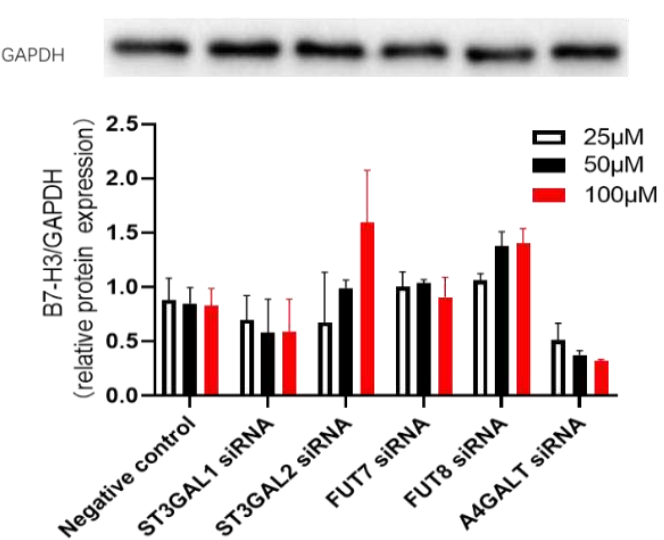
Figure 3

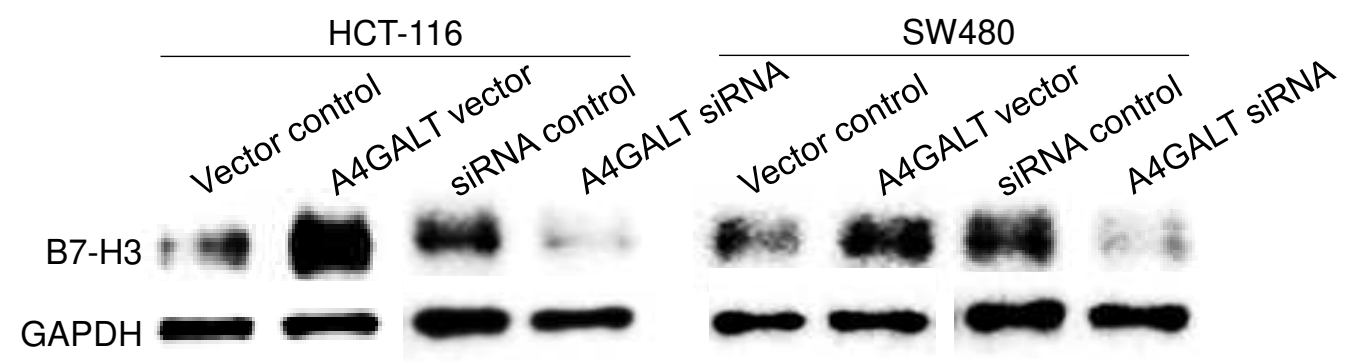


Figure 4

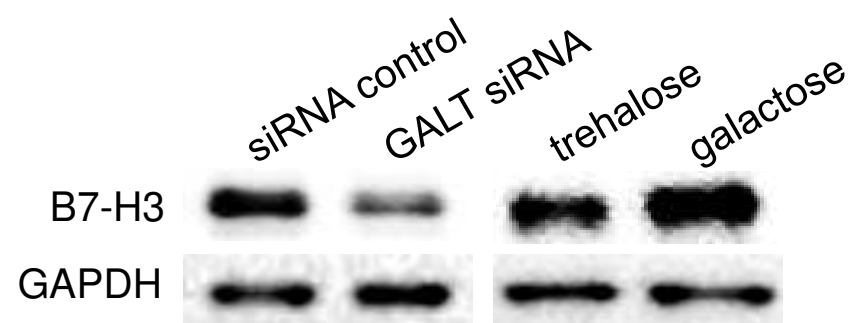


Figure 5

$\begin{array}{rrrrrr}\text { B7-H3 antibody } & - & & - & + & \\ \text { GAPDH antibody } & - & & + & - & \\ \text { Protein a agarose } & + & & + & + & \text { Input } \\ \text { B7-H3 } & & & & & \\ \text { A4GALT } & & & & \end{array}$




\section{Supplementary Files}

This is a list of supplementary files associated with this preprint. Click to download.

- SupplementaryMaterials20211206.docx 